\title{
The longitudinal association between weight change and health-related quality of life in adults and
}

\section{children: a systematic review}

Mark Hayes $^{1,2}$, Helen Baxter ${ }^{3}$, Jacqueline Müller-Nordhorn ${ }^{1}$, Johanna Katharina Hohls $^{1,4}$, Rebecca Muckelbauer ${ }^{1}$

${ }^{1}$ Institute of Public Health, Charité - Universitätsmedizin Berlin, Berlin, Germany

${ }^{2}$ The University of Melbourne, Melbourne Medical School, Melbourne, VIC, Australia

${ }^{3}$ Austin Health Sciences Library, Austin Health, Heidelberg, VIC, Australia

${ }^{4}$ Department of Health Economics and Health Services Research, University Medical

Centre Hamburg-Eppendorf, Hamburg, Germany

\section{Key Words}

Weight gain; weight loss; body mass index; obesity; health-related quality of life

\section{Running Title}

Weight change and health-related quality of life

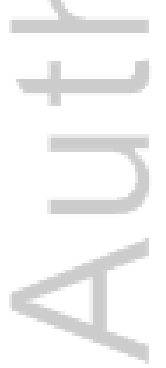

This is the author manuscript accepted for publication and has undergone full peer review but has not been through the copyediting, typesetting, pagination and proofreading process, which may lead to differences between this version and the Version of Record. Please cite this article as doi: 10.1111/obr.12595

This article is protected by copyright. All rights reserved. 


\section{Acknowledgements}

Mark Hayes was funded through scholarships provided by the German Academic Exchange Service and the University of Melbourne (John and Betty McCreery Scholarship and the Alan Gilbert Mobility Scholarship).

\section{Contact details:}

Mark Hayes

1/58 Arthur St

Fairfield, VIC, 3078

Australia

markjosephhayes@gmail.com

Conflicts of interest: Mark Hayes was funded through scholarships provided by the German Academic Exchange Service and the University of Melbourne (John and Betty McCreery Scholarship and the Alan Gilbert Mobility Scholarship).

Johanna Hohls' contract was paid for using finances supplied by the University of Melbourne to Charité.

This article is protected by copyright. All rights reserved. 


\section{Abstract}

This systematic review examined longitudinal associations between weight change (weight gain and loss) and both physical and mental aspects of health-related quality of life (HRQOL) compared with stable weight in adults and children of the general population. MEDLINE, EMBASE, PsycINFO, and PubMed databases were searched. Longitudinal observational studies measuring $\mathrm{HRQOL}$ with six predefined instruments were synthesised according to type of association: weight change and change in HRQOL (change-on-change association); and weight change and HRQOL at follow-up (predictive association). Twenty studies of adults $(n=15)$ or children $(n=$ 5) were included. 15 studies used the SF-12 or SF-36. Results of nine studies in adults examining the change-on-change association were combined through a tallying of 606 analyses. Weight gain was most often associated with reduced physical, but not mental HRQOL, across all baseline body mass index categories and in both males and females. Weight loss may be associated with improved physical, but not mental HRQOL, among adults with overweight and obesity. Weight gain was more strongly associated with $\mathrm{HRQOL}$ than weight loss, implicating a greater need for preventative strategies to tackle obesity. Results in children and for the predictive association generally reflected these findings, but require further research.

This article is protected by copyright. All rights reserved. 


\section{Introduction}

Since 1980 , the prevalence of overweight and obesity has increased worldwide by $28 \%$ among adults and $47 \%$ among children. ${ }^{1}$ This global epidemic is a major public health priority, with universal concern regarding the health risks and associated costs and lost productivity. ${ }^{2}$

Overweight and obesity are associated with elevated morbidity and mortality risks across the lifespan ${ }^{3,4}$, in addition to reduced health-related quality of life (HRQOL). ${ }^{5}$ HRQOL is a multidimensional concept that focuses on the impact of a disease or health state on an individual's physical, mental, and social wellbeing. ${ }^{6}$ Low HRQOL has been shown to predict premature mortality, some morbidities, and health service utilisation. $^{7-9}$

Systematic reviews and meta-analyses of cross-sectional studies show that a higher than normal body mass index (BMI) in adults (defined as $>25 \mathrm{~kg} / \mathrm{m}^{2}$ ) and children (defined in individual studies by age and sex specific centile cut-offs) is associated with lower physical HRQOL, with strong evidence of a dose-responsive relationship. ${ }^{10-14}$ Associations with mental HRQOL tended to be weaker or less consistent. ${ }^{10-14}$ These results, however, are unable to demonstrate any longitudinal relationship between weight changes and HRQOL. It is reasonable to hypothesise 
that weight gain over the lifespan would lower physical HRQOL, while weight loss would improve physical HRQOL.

In a systematic review and meta-analysis of randomised controlled trials for weight loss, Warkentin et al ${ }^{15}$ showed intentional weight loss is associated with improved physical, but not with overall or mental HRQOL. A systematic review of weight loss interventions by Kroes et $\mathrm{al}^{16}$ further found a direct dose-responsive relationship, with greater degrees of weight loss correlating with greater improvements in physical HRQOL. The review found only one study reporting a significant correlation between weight loss and improved mental HRQOL after surgery, with the other studies showing no correlation. ${ }^{16}$ Exposure to the weight loss intervention, rather than to weight loss itself, may partly mediate these HRQOL improvements. ${ }^{17-20}$ Moreover, the results of weight loss studies are not transferable to the general population with or without obesity, as people with obesity seeking weight loss treatment have lower HRQOL thank the general population with obesity. ${ }^{21}$

Longitudinal observational studies offer the potential to examine the relationship between weight changes and HRQOL in the general population without confounding effects from interventions. The primary objective of this systematic review of published observational studies was to determine if exposure to weight changes (weight gain or loss) in adults and children with and without obesity is longitudinally associated with HRQOL outcomes, compared with stable weight. 


\section{Methods}

We conducted a systematic review registered with PROSPERO, the International Prospective Register for Systematic Reviews (http://www.crd.york.ac.uk/prospero/, registration number CRD42016027434).

\section{Search strategy}

We searched the following databases on $28^{\text {th }}$ January 2016: MEDLINE (Ovid)(19462016), EMBASE (Ovid)(1947-2016), PsycINFO (Ovid)(1806-2016) and PubMed (1809-2016), with an updated search conducted on $28^{\text {th }}$ July 2016 . The MEDLINE search strategy is displayed in Table 1 and translated strategies for other databases are available in the Supplementary Material Table S1. To minimise duplication, we utilised EMBASE and PubMed search options to exclude studies already indexed in MEDLINE. Full citations were exported into the referencing software EndNote $X 7$ for storage, management and removal of duplicates. One author $(\mathrm{MJH})$ sought further relevant studies by hand searching references and contacting authors of included studies. He additionally applied PubMed 'cited by' and 'similar articles' tools to all included studies. All records identified by the former were retrieved, while for the latter the first 10 records by relevance were retrieved.

\section{Study selection}

All titles and abstracts from the initial electronic search were screened by the primary reviewer $(\mathrm{MJH})$ for inclusion. Full texts of included studies and references of included systematic reviews were then screened against eligibility criteria. To increase the quality of the review by ensuring the accuracy of the primary reviewer, a second 
reviewer $(\mathrm{KJH})$ independently screened half of the titles and abstracts for inclusion and subsequently performed full text screening. Reviewers were blinded to studies' authors during the initial screen of titles and abstracts. Inter-rater agreement was assessed by Cohen's kappa co-efficient calculated using Stata version 13.0 (Stata Corporation, College Station, TX). ${ }^{22}$ Discrepancies between reviewers were resolved through discussion with a third reviewer (RM).

This article is protected by copyright. All rights reserved. 
Table 1 Search strategy for MEDLINE via Ovid

\section{Nr. Search Term}

1 exp "Quality of Life"/

2 ((life adj2 qualit\$) or QOL\$ or HRQL or HRQOL or SF12 or SF-12 or short form 12 or SF-36 or SF36 or short form 36 or Euroqol or EQ-5D or EQ5D or (Pediatric Quality adj Life Inventory) or PedsQL or KIDSCREEN or Child Health Questionnaire or CHQ).tw.

31 or 2

4 exp Body Composition/ or exp Overweight/ or Body Weight/ or "Body Weights and Measures"/ or Thinness/ or Body Mass Index/ or Body Size/ or Waist Circumference/ or Waist-Hip Ratio/ or WaistHeight ratio/ or Weight Gain/ or Weight Loss/ or Skinfold Thickness/ or Pediatric Obesity/ or Growth Charts/ or Adipose Tissue/

5 (body weight or body mass or body size or body fat or body composition or abdominal fat or obesity or obese or overweight or thinness or underweight or fatty tissue or adipos $\$$ or body measure $\$$ or bmi or waist circumference or waist hip ratio or weight height ratio or height weight ratio or waist height ratio or skinfold thickness or weight gain or weight increase or weight change or weight loss or weight reduction or weight decrease or growth chart\$ or weight centile or weight percentile).tw.

64 or 5

7 exp Cohort Studies or Case-control Studies/ or Epidemiologic Studies/

8 (prospective or retrospective or longitudinal or observational or follow up or cohort or case control).tw.

97 or 8

$10 \quad 3$ and 6 and 9

1110 and (exp animals/ or exp veterinary medicine/ or exp animal experimentation/)

1210 and humans/

1311 not 12

1410 not 13

1514 not (case reports or editorial or in vitro).pt.

16 limit 15 to (english or french or german or spanish)

This article is protected by copyright. All rights reserved. 


\section{Eligibility criteria}

Longitudinal observational studies reporting an association between weight change and $\mathrm{HRQOL}$ outcomes and of at least one year follow-up were included, as we considered this the minimum duration for meaningful changes in weight or HRQOL. To reduce publication bias, we sought studies published in all languages known to the authors (English, French, German and Spanish). Cross-sectional studies, case reports, editorials, conference proceedings and abstracts, and primary intervention studies for weight loss were excluded, as they either provide no longitudinal data; are of low evidence level; or, in the case of the latter, introduce the potential for confounding by the intervention. Secondary analyses of intervention studies were considered if the analyses were presented for the control group separately or if exposure to the intervention was shown not to, or could not impact independently on weight and HRQOL.

Studies were included if they reported subscales, component summary scores or overall HRQOL using the Short Form- $36^{23}$ (SF-36), Short Form-12 ${ }^{24}$ (SF-12), or Euroqol 5D ${ }^{25}$ (EQ-5D) in adults or the Pediatric Quality of Life Inventory ${ }^{26}$ (PedsQL), Child Health Questionnaire ${ }^{27}$, or KIDSCREEN ${ }^{28}$ in children. These instruments were selected for inclusion because of their frequent use and demonstrated validity. ${ }^{29}, 30$ To ensure data completeness, studies reporting fewer than half of an instrument's subscales were excluded. We included studies with participants of all weight categories, including underweight $\left(\mathrm{BMI}<18.5 \mathrm{~kg} / \mathrm{m}^{2}\right)$. 
Studies were excluded if they analysed only hospitalised or pregnant participants or participants all having a disease or condition (other than overweight or obesity), or receiving a specific treatment, as these variables may confound the relationship between weight change and HRQOL. For studies analysing the same sample and conducting the same analysis but at different follow-up times, only the most recent or complete study was included to avoid data duplication. Studies analysing the same participants answering different research questions were included as separate studies.

\section{Data extraction}

Data of included studies was extracted by one reviewer $(\mathrm{MJH})$. Extracted data included: study details (authors, year of publication, location); participant characteristics (age range and/or mean and standard deviation, sex distribution, population source); primary aim of the study; methods (design, assessment of body weight and HRQOL, statistical methods applied); results for analyses between weight change and HRQOL; and sources of funding. For analyses treating weight change as a categorical variable, we extracted results for two categories: dichotomised as weight gain and weight loss, each compared with weight stable. Weight stable groups were those with no or minimal weight change, as defined in respective studies. If weight change was a continuous variable, it was not possible to distinguish between weight gain and weight loss and we extracted the linear measure of association. For studies providing separate analysis for participants with specific diseases or exposed to an intervention impacting independently on weight or HRQOL, only data on 
participants without the disease or intervention were extracted. If required, first and/or last study authors study were contacted to retrieve any missing data or unreported information. Significance of all associations was inferred from statistical tests or confidence intervals, with $\mathrm{p}<0.05$ considered significant unless otherwise stated.

\section{Synthesis of results}

Variable treatment of weight change data precluded a meta-analysis. Instead we performed a narrative synthesis of study results, focused on the direction of association of weight changes with HRQOL, rather than effect sizes. Results were summarised in a table, stratified by both age group (adults: baseline age $\geq 18$ years, children: baseline age $<18$ years) and type of longitudinal association. Two types of longitudinal associations were examined: the longitudinal relationship between weight change and change in HRQOL between baseline and follow-up (change-onchange association); and the longitudinal relationship between weight change and HRQOL at follow-up without adjustment for baseline HRQOL (predictive association). Studies associating weight change and HRQOL at follow-up with adjustment for baseline HRQOL were interpreted as belonging to the change-on-change association.

Where HRQOL was measured by SF-36, SF-12 or PedsQL, results for physical and mental/psychosocial component summary scores, or for instrument subscales were included. For SF-36 and SF-12, subscales of physical functioning, physical role limitation, bodily pain, and general health relate to physical HRQOL, while subscales of social functioning, emotional role limitation, vitality, and mental health relate to 
mental HRQOL. ${ }^{23,24}$ For other HRQOL instruments, results for either the overall scores or subscales were included.

\section{Tallying synthesis}

A subset of studies treating weight change as a categorical variable produced many results, which could not be easily summarised through narrative synthesis. To summarise these results, we tallied the number of analyses from these studies according to the direction of weight change experienced (gain or loss) and the resulting change in HRQOL subscales or component summary scores. All categorical change-on-change results in adults were combinable through a tallying synthesis, as all studies used the SF-36 or SF-12. Tallied analyses were presented graphically in vertical bar charts categorised by direction (reduced, improved, or neither reduced nor improved) and statistical significance (significant or non-significant) of associations. Analysed, but non-reported results were combined with the nonsignificant associations. We also presented the tallied analyses stratified by sex (male and female) and baseline BMI category (normal weight, BMI $<25 \mathrm{~kg} / \mathrm{m}^{2}$; overweight $\mathrm{BMI}=25-29.9 \mathrm{~kg} / \mathrm{m}^{2}$; and obese, $\mathrm{BMI}>30 \mathrm{~kg} / \mathrm{m}^{2}$ ).

\section{Quality and risk-of-bias assessment}

Two reviewers ( $\mathrm{MJH}$ and $\mathrm{JKH})$ independently assessed the quality of included studies as poor, fair or good using the Quality Assessment Tool for Observational Cohort and Cross-Sectional Studies (QATOCCS). ${ }^{31}$ Discrepancies between reviewers were resolved through discussion with a third reviewer (RM). 


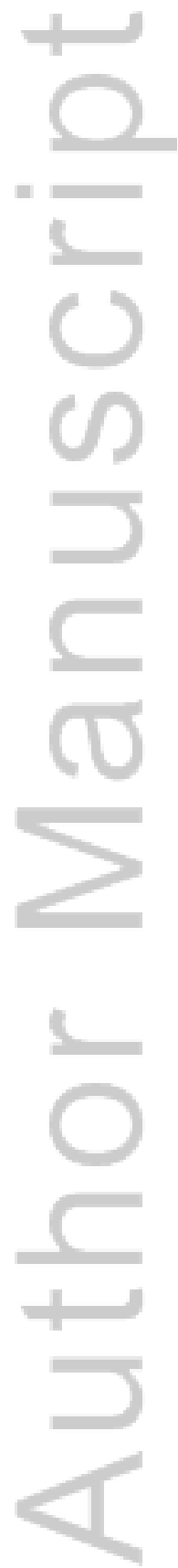

This article is protected by copyright. All rights reserved. 


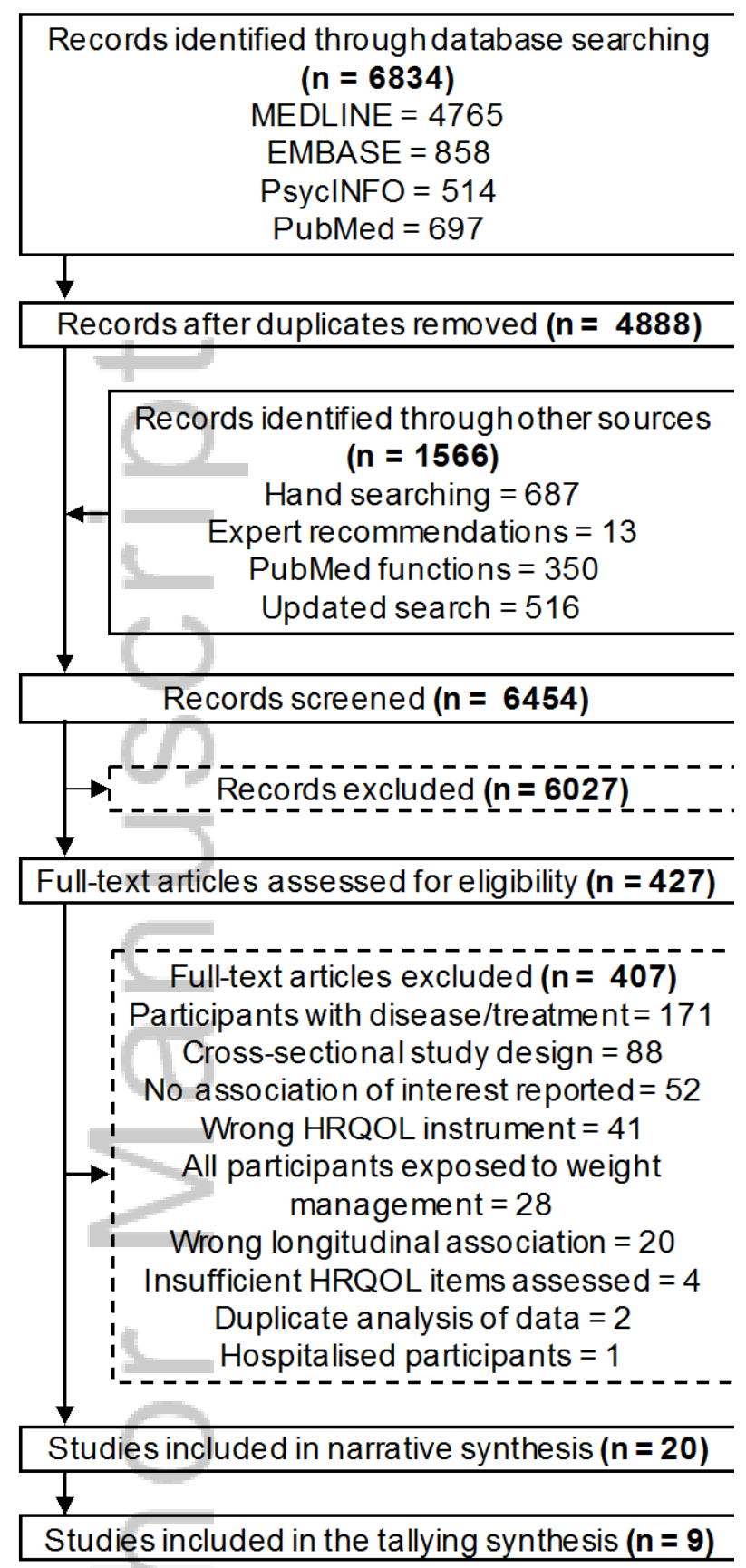

Figure 1 Results of search and screening process from the identification to the inclusion of studies, based on PRISMA flow diagram ${ }^{32}$

This article is protected by copyright. All rights reserved. 
Table 2 Characteristics of included studies separately for adults and children, according to the longitudinal association analysed

\begin{tabular}{|c|c|c|c|c|c|c|c|c|c|c|}
\hline $\begin{array}{l}\text { Age } \\
\text { group }\end{array}$ & $\begin{array}{l}\text { Association } \\
\text { type }\end{array}$ & Reference & $\begin{array}{l}\text { Follow- } \\
\text { up } \\
\text { (years) }\end{array}$ & Country & $\begin{array}{l}\text { Sample } \\
\text { size }\end{array}$ & $\begin{array}{l}\text { Sex } \\
(\% \mathrm{~F})\end{array}$ & $\begin{array}{l}\text { Age range } \\
\text { or mean } \\
\text { (SD) }\end{array}$ & $\begin{array}{l}\text { Weight change exposure } \\
\text { (categories) }^{\mathrm{a}}\end{array}$ & $\begin{array}{l}\text { Weight stable } \\
\text { comparator } \\
\text { (categories) }\end{array}$ & $\begin{array}{l}\text { HRQOL outcome } \\
\text { (items analysed) }\end{array}$ \\
\hline \multirow[t]{13}{*}{ Adults } & $\begin{array}{l}\text { Change-on- } \\
\text { change }\end{array}$ & Batsis $^{33} 2015$ & 6 & USA & 664 & $\begin{array}{l}\text { F\&M } \\
(61.1)\end{array}$ & $67.9(5.3)$ & $\begin{array}{l}\text { WG }(\geq 5 \%) \\
\text { WL }(\geq 5 \%)\end{array}$ & $\Delta \mathrm{W}< \pm 5 \%$ & $\begin{array}{c}\Delta S F-12 \\
(\mathrm{PCS} / \mathrm{MCS})\end{array}$ \\
\hline & & Cameron $^{34} 2012$ & 5 & Australia & 5,985 & $\begin{array}{l}\text { F\&M } \\
(54.7)\end{array}$ & $51.3(4.5)$ & WG $\left(>0 \mathrm{~kg} / \mathrm{m}^{2}\right)$ & $\Delta \mathrm{W} \leq 0 \mathrm{~kg} / \mathrm{m}^{2}$ & $\begin{array}{c}\Delta S F-36 \\
(\mathrm{PCS} / \mathrm{MCS})\end{array}$ \\
\hline & & de Hollander 2013 & 15 & $\begin{array}{l}\text { the } \\
\text { Netherlands }\end{array}$ & 3408 & $\begin{array}{l}F \& M \\
(56.5)\end{array}$ & $24-66$ & WG $(\mathrm{NW} \rightarrow \mathrm{OW} ; \mathrm{NW} / \mathrm{OW} \rightarrow \mathrm{O})$ & WS $(\mathrm{NW} \rightarrow \mathrm{NW})$ & $\begin{array}{c}\Delta S F-36^{b} \\
\text { (8 subscales) }\end{array}$ \\
\hline & & Fine $^{36} 1999$ & 4 & USA & 40,098 & $\begin{array}{c}F \\
(100)\end{array}$ & $46-71$ & $\begin{array}{l}W^{c}(2.25-9 \mathrm{~kg} ; \geq 9 \mathrm{~kg}) \\
W^{c}(2.25-9 \mathrm{~kg} ; \geq 9 \mathrm{~kg})\end{array}$ & $\Delta \mathrm{W}^{\mathrm{C}}< \pm 2.25 \mathrm{~kg}$ & $\begin{array}{c}\Delta \text { SF-36 } \\
\text { (7 subscales) }\end{array}$ \\
\hline & & Herpetz $^{37} 2015$ & 9 & $\begin{array}{l}\text { Germany } \\
\text { Austria }\end{array}$ & 83 & $\begin{array}{l}\text { F\&M } \\
(71.9)\end{array}$ & $\begin{array}{r}42.5 \\
(11.8)\end{array}$ & $\Delta \mathrm{BMI}$ (continuous) & NA & $\begin{array}{c}\triangle \mathrm{SF}-36 \\
(\mathrm{PCS} / \mathrm{MCS})\end{array}$ \\
\hline & & Laxy $^{38} 2014$ & 7 & Germany & 3080 & $\begin{array}{l}\text { F\&M } \\
(51.8)\end{array}$ & $25-74$ & $\begin{array}{l}\text { 1. WG }(5-10 \% ;>10 \%) \\
\text { 1. WL }(5-10 \% ;>10 \%)\end{array}$ & 1. $\Delta \mathrm{W}< \pm 5 \%$ & $\begin{array}{c}\triangle \mathrm{SF}-12 \\
(\mathrm{PCS} / \mathrm{MCS})\end{array}$ \\
\hline & & León-Muñoz 2005 & 2 & Spain & 2364 & $\begin{array}{l}\text { F\&M } \\
(56.4)\end{array}$ & $60-93$ & $\begin{array}{l}\text { 2. } \triangle \mathrm{BMI} \text { (continuous) } \\
\mathrm{WG}^{\mathrm{C}} \\
\mathrm{WL}^{\mathrm{C}}\end{array}$ & $\begin{array}{c}\text { 2. NA } \\
\Delta \mathrm{W}^{\mathrm{c}}=\text { no }\end{array}$ & $\begin{array}{c}\Delta \text { SF-36 } \\
\text { (8 subscales) }\end{array}$ \\
\hline & & Milder $^{40} 2014$ & 5 & $\begin{array}{l}\text { the } \\
\text { Netherlands }\end{array}$ & 4,135 & $\begin{array}{l}\text { F\&M } \\
(51.5)\end{array}$ & $26-70$ & $\begin{array}{c}\text { WG }(2.1-4 \mathrm{~kg} ; 4.1-6 \mathrm{~kg} ;>6 \mathrm{~kg}) \\
W L(>2 \mathrm{~kg})\end{array}$ & $\Delta \mathrm{W}< \pm 2 \mathrm{~kg}$ & $\begin{array}{c}\Delta \text { SF- } 36^{\mathrm{b}} \\
\text { (8 subscales) }\end{array}$ \\
\hline & & $\operatorname{Pan}^{41} 2014$ & 4 & USA & 105,269 & $\begin{array}{c}F \\
(100)\end{array}$ & 29-71 & $\begin{array}{l}W^{c, d}(2.25-6.74 \mathrm{~kg} ; \geq 6.75 \mathrm{~kg}) \\
W^{\mathrm{c}, \mathrm{d}}(2.25-6.74 \mathrm{~kg} ; \geq 6.75 \mathrm{~kg})\end{array}$ & $\Delta \mathrm{W}^{\mathrm{c}}< \pm 2.25 \mathrm{~kg}$ & $\begin{array}{c}\triangle \mathrm{SF}-36 \\
(\mathrm{PCS} / \mathrm{MCS})\end{array}$ \\
\hline & & Williams $^{42} 2006$ & 2 & Australia & 7270 & $\begin{array}{c}F \\
(100)\end{array}$ & $45-50$ & $\begin{array}{c}W G^{c}(2.25-4.4 \mathrm{~kg} ; \geq 4.5 \mathrm{~kg}) \\
W L(\geq 2.25 \mathrm{~kg})\end{array}$ & $\Delta \mathrm{W}^{\mathrm{C}}< \pm 2.25 \mathrm{~kg}$ & $\begin{array}{c}\Delta S F-36 \\
(\mathrm{PCS} / \mathrm{MCS})\end{array}$ \\
\hline & ictive & Burns $^{43} 2001$ & 5 & $\begin{array}{l}\text { the } \\
\text { Netherlands }\end{array}$ & 1113 & $\begin{array}{l}\text { F\&M } \\
(52.0)\end{array}$ & $26-59$ & $\begin{array}{l}\text { WG }(5-10 \% ;>10 \%) \\
\text { WL (5-10\%; >10\%) }\end{array}$ & $\Delta \mathrm{W}< \pm 5 \%$ & $\begin{array}{c}\text { SF- } 36^{\mathrm{b}} \\
\text { (8 subscales) }\end{array}$ \\
\hline & & Connor $^{44} 2016$ & $\sim 14.5$ & USA & 270 & $\begin{array}{c}F \\
(100)\end{array}$ & $30-74$ & $\Delta \mathrm{BMI}^{\mathrm{C}}$ (continuous) & NA & $\begin{array}{c}\text { SF-12 } \\
\text { (PCS/MCS) }\end{array}$ \\
\hline & 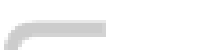 & Döring $^{45} 2015$ & 8 & Sweden & 16,666 & F\&M & $18-84$ & $W^{c}{ }^{c}(5.1-9.9 \% ; \geq 10 \%)$ & $\Delta \mathrm{W}^{\mathrm{C}} \leq \pm 5 \%$ & EQ-5D \\
\hline
\end{tabular}

This article is protected by copyright. All rights reserved. 


\begin{tabular}{|c|c|c|c|c|c|c|c|c|c|}
\hline 1 & Kozak $^{46} 2011$ & 20 & USA & 3014 & $\begin{array}{c}(56.6) \\
F \& M \\
(55.0)\end{array}$ & $18-30$ & $\begin{array}{c}W^{\mathrm{C}}(5.1-9.9 \% ; \geq 10 \%) \\
\text { 1. WG }(\mathrm{NW} \rightarrow \mathrm{OW} ; \mathrm{NW} \rightarrow \mathrm{O}) \\
\text { 2. WG }(\mathrm{OW} \rightarrow \mathrm{O})\end{array}$ & $\begin{array}{l}\text { 1. WS }(\mathrm{NW} \rightarrow \mathrm{NW}) \\
\text { 2. WS }(\mathrm{OW} \rightarrow \mathrm{OW})\end{array}$ & $\begin{array}{c}\text { SF-12 } \\
\text { (PCS/MCS) }\end{array}$ \\
\hline & Strandberg $^{47} 2003$ & 26 & Finland & 1147 & $\begin{array}{c}M \\
(0.0)\end{array}$ & $\begin{array}{c}39-56 \\
(47)\end{array}$ & $\begin{array}{c}W^{c, ~ e+}(0.1-4.0 k g ; 4.1- \\
9.0 k g ; 9.1-14.9 k g ; \geq 15 k g)\end{array}$ & $\Delta \mathrm{W}^{\mathrm{C}} \leq \mathrm{Okg}$ & $\begin{array}{c}\text { SF-36 } 6^{\mathrm{b}} \\
\text { (8 subscales) }\end{array}$ \\
\hline
\end{tabular}

Table 2 Continued.

\begin{tabular}{|c|c|c|c|c|c|c|c|c|c|c|}
\hline $\begin{array}{l}\text { Age } \\
\text { group }\end{array}$ & $\begin{array}{l}\text { Association } \\
\text { type }\end{array}$ & Reference & $\begin{array}{l}\text { Follow- } \\
\text { up } \\
\text { (years) }\end{array}$ & Country & $\begin{array}{l}\text { Sample } \\
\text { size }\end{array}$ & $\begin{array}{l}\text { Sex } \\
(\% \mathrm{~F})\end{array}$ & $\begin{array}{l}\text { Age range } \\
\text { or mean } \\
\text { (SD) }\end{array}$ & $\begin{array}{l}\text { Weight change exposure } \\
\text { (categories) }\end{array}$ & $\begin{array}{c}\text { Weight stable } \\
\text { comparator } \\
\text { (categories) }\end{array}$ & $\begin{array}{l}\text { HRQOL outcome } \\
\text { (items analysed) }\end{array}$ \\
\hline \multirow[t]{5}{*}{ Childre } & $\begin{array}{l}\text { Change-on- } \\
\text { change }\end{array}$ & Sawyer $^{48} 2011$ & 4 & Australia & 3363 & $\begin{array}{l}F \& M \\
(49.0)\end{array}$ & $4-5$ & $\Delta \mathrm{BMI}$ Z-score (continuous) & NA & $\begin{array}{c}\text { PedsQL } \\
\text { (PCS/MCS, } \\
\text { subscales) }\end{array}$ \\
\hline & Predictive & Gopinath $^{49} 2013$ & 5 & Australia & 1025 & $\begin{array}{l}F \& M \\
(52.6)\end{array}$ & $12-13$ & $\mathrm{WL}(\mathrm{OW} / \mathrm{O} \rightarrow \mathrm{NO})$ & WS $(\mathrm{O} \rightarrow \mathrm{O})$ & $\begin{array}{c}\text { PedsQL } \\
\text { (PCS/MCS, } \\
\text { subscales) }\end{array}$ \\
\hline & & Herman $^{50} 2010$ & 22 & Canada & 310 & $\begin{array}{l}\text { F\&M } \\
(50.6)\end{array}$ & $7-18$ & $\mathrm{WG}^{\mathrm{c}}(\mathrm{NW} \rightarrow \mathrm{OW} / \mathrm{O})$ & $\mathrm{WS}^{\mathrm{c}}(\mathrm{NW} \rightarrow \mathrm{NW})$ & SF-36 (subscales) \\
\hline & & Parkinson $^{51} 2015$ & $2-5$ & UK & $\begin{array}{c}331- \\
445\end{array}$ & $\begin{array}{l}\text { F\&M } \\
(51.0)\end{array}$ & $6-8$ & $\begin{array}{l}\Delta \mathrm{FMI} \text { (continuous) } \\
\Delta \mathrm{WC} \text { (continuous) }\end{array}$ & NA & $\begin{array}{l}\text { KIDSCREEN-27 } \\
\text { subscales }\end{array}$ \\
\hline & & Wake $^{52} 2010$ & 8 & Australia & 932 & $\begin{array}{l}F \& M \\
(49.4)\end{array}$ & $5-10.7$ & WG $(\mathrm{NW} \rightarrow \mathrm{OW} / \mathrm{O})$ & $(\mathrm{NW} \rightarrow \mathrm{NW})$ & $\begin{array}{c}\text { PedsQL } \\
\text { (PCS/MCS) }\end{array}$ \\
\hline
\end{tabular}

Abbreviations: $\Delta$, change; BMI, body mass index; CD, cannot determine; EQ-5D, Euroqol 5D; FMI, fat mass index; F, female; HRQOL, health-related quality of life; M, male; MCS, mental/psychosocial component summary score; NA, not applicable; NR, not reported; NW, normal weight; O, obese; OW, overweight; PCS, physical component summary score; PedsQL, Pediatric Quality of Life Inventory; SF-12, Short Form 12; SF-36, Short Form 36;

WC, waist circumference; WG, weight gain; WL, weight loss; WS, weight stable; $\Delta \mathrm{W}$, weight change

${ }^{\mathrm{a}}$ The symbol $\rightarrow$ represents transition from one weight category to another.

${ }^{b}$ SF-36 scores calculated using the RAND-36 calculation methodology. ${ }^{53}$

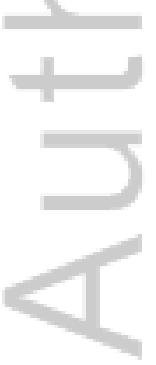

This article is protected by copyright. All rights reserved. 
${ }^{c}$ Measured by self-report

${ }^{\mathrm{d}}$ Measurement converted from pounds, based on the authors provided conversion ratio $(1 \mathrm{lb}=0.45 \mathrm{~kg})$

e Baseline weight measurement was retrospective (recalled weight at age at 25 years)

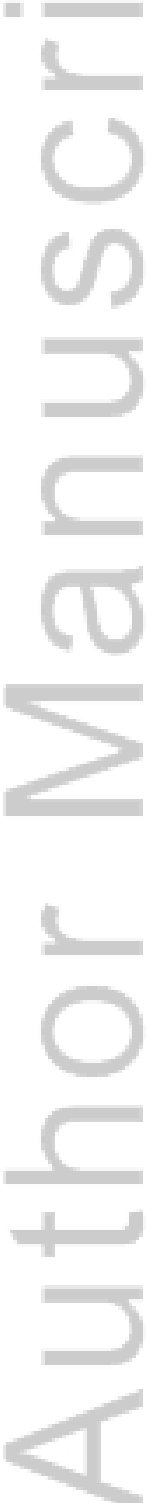

This article is protected by copyright. All rights reserved. 
Table 3 Main results from included studies on the association between direction of weight change and HRQOL outcomes and the number of analysis contributing the tallied synthesis.

\begin{tabular}{|c|c|c|c|c|c|c|c|c|}
\hline \multirow{2}{*}{$\begin{array}{l}\text { Age } \\
\text { group }\end{array}$} & Association & \multirow[t]{2}{*}{ Reference } & \multicolumn{2}{|c|}{ Weight gain } & \multicolumn{2}{|c|}{ Weight loss } & \multicolumn{2}{|c|}{ Weight change $^{a}$} \\
\hline & type & & Physical & Mental & Physical & Mental & Physical & Mental \\
\hline \multirow[t]{14}{*}{ Adults } & Change-on- & Batsis $^{33} 2015$ & 1 & 1 & 1 & 1 & & \\
\hline & change & Cameron $^{34} 2012$ & 6 & 6 & & & & \\
\hline & & de Hollander ${ }^{35} 2013$ & 16 & 16 & & & & \\
\hline & & Fine $^{36} 1999$ & 48 & 64 & 48 & 64 & & \\
\hline & & Herpetz $^{37} 2015$ & & & & & $\begin{array}{l}\text { Significant, } \\
\text { direction not } \\
\text { reported }\end{array}$ & Non-significant \\
\hline & & $\operatorname{Laxy}^{38} 2014$ & 12 & 12 & 12 & 12 & $\begin{array}{c}\text { Inverse } \\
\text { association with } \\
\text { PCS }\end{array}$ & $\begin{array}{l}\text { Direct association } \\
\text { with MCS }\end{array}$ \\
\hline & & León-Muñoz 2005 & 16 & 16 & 16 & 16 & & \\
\hline & & Milder $^{40} 2014$ & 72 & 72 & 24 & 24 & & \\
\hline & & $\operatorname{Pan}^{41} 2014$ & 6 & 6 & 6 & 6 & & \\
\hline & & Williams $^{42} 2006$ & 2 & 2 & 1 & 1 & & \\
\hline & edictive & Burns $^{43} 2001$ & $\begin{array}{l}\text { Lower physical } \\
\text { functioning and } \\
\text { general health } \\
\text { subscales }\end{array}$ & $\begin{array}{l}\text { Lower vitality } \\
\text { subscale }\end{array}$ & Non-significant & Non-significant & & \\
\hline & & Connor $^{44} 2016$ & & & & & Non-significant & Non-significant \\
\hline & & Döring ${ }^{45} 2015$ & $\begin{array}{l}\text { Lower overall EQ- } \\
\text { 5D in all BMI } \\
\text { categories }\end{array}$ & $\begin{array}{l}\text { Lower overall EQ- } \\
\text { 5D in all BMI } \\
\text { categories }\end{array}$ & $\begin{array}{l}\text { Lower overall EQ- } \\
5 D \text { in normal } \\
\text { weight and } \\
\text { overweight }^{b}\end{array}$ & $\begin{array}{l}\text { Lower overall EQ- } \\
\text { 5D in normal } \\
\text { weight and } \\
\text { overweight }^{b}\end{array}$ & & \\
\hline & & $\begin{array}{l}\text { Kozak }^{46} 2011 \\
\text { Strandberg }^{41} 2003\end{array}$ & $\begin{array}{c}\text { Lower PCS } \\
\text { Lower on all } \\
\text { physical subscales }\end{array}$ & $\begin{array}{l}\text { Non-significant } \\
\text { Lower mental } \\
\text { subscales, except } \\
\text { mental health }\end{array}$ & & & & \\
\hline
\end{tabular}


Children Change-on- Sawyer ${ }^{48} 2011$ change

$=$ Predictive Gopinath ${ }^{49} 2013$

Herman $^{50} 2010$

Parkinson $^{51} 2015$

Wake $^{52} 2010$

health subscale

Lower PCS
Inverse

association with

PCS

Higher PCS in

obese

Non-significant

Lower MCS

Non-significant ${ }^{\mathrm{b}}$

Non-significant ${ }^{\mathrm{b}}$

EQ-5D, Euroqol 5D; MCS, mental/psychosocial component summary score; PCS, physical component summary score; TS, tallied synthesis

Weight change as a continuous variable

${ }^{b}$ Significance was set at the $p<0.01$ level

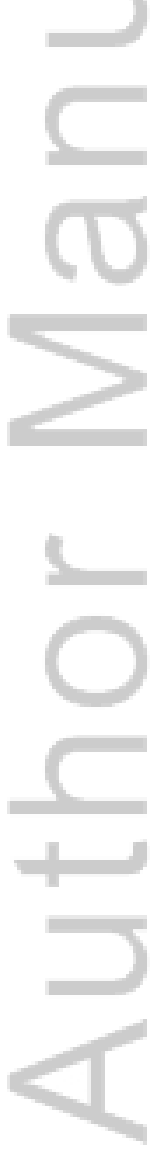

This article is protected by copyright. All rights reserved. 


\section{Results}

Figure 1 shows the study selection process. One reviewer screened titles and abstracts of 4888 articles identified from the electronic search and 1566 from other sources. From these, 427 were selected for full text screening, which identified 20 studies meeting the eligibility criteria for inclusion in our review. To increase the quality of the review, a second reviewer additionally screened a sample of 2444 articles independently. The Cohen's kappa coefficient for was 0.799 , representing very substantial agreement between the two reviewers. ${ }^{54}$

Characteristics of included studies are summarised in Table 2. All studies were cohort studies, with follow-up times ranging from two to 26 years. Fifteen studies were in adults and five in children. Eleven studies analysed the change-on-change association, while nine analysed the predictive association. Fifteen studies analysed weight change exclusively as a categorical variable; four studies exclusively as a continuous variable; and one study as both. Anthropometric data was measured by self-report in eight studies and by physical examination in twelve. In adults, ten studies used SF-36; four studies used SF-12; and one study used EQ-5D. In children, three studies used PedsQL; one study used SF-36; and one study used KIDSCREEN-27.

Further study characteristics, including data source, statistical methods, quality and risk of bias assessment results are summarised in Supplementary Material Table S2. Of note, two studies sourced data from the Nurses Health Study ${ }^{36,41}$ and a further 
two studies from the Doetinchem Cohort Study. ${ }^{35,40}$ Two studies included underweight participants within the normal weight category, the remaining studies excluded underweight participants. ${ }^{36,40}$ According to the QATOCCs quality assessment instrument, two of the included studies were rated as good ${ }^{35,40} ; 17$ as fair $^{33,34,36-39,41-43,45-52}$; and one as poor quality. ${ }^{44}$

Of the studies treating weight change as a categorical variable, nine studies in adults examining the change-on-change association contributed to the tallying synthesis. ${ }^{33-}$

42 These nine studies provided 606 analyses of the association between weight change and change in HRQOL, with Table 3 summarising the number of analyses contributed by each study. ${ }^{33-42}$

\section{Weight gain}

\section{Adults}

Results for weight gain in adults via the change-on-change association are summaries in Figure 2A. Overall, from nine studies there were 374 analyses of the association between weight gain and change in HRQOL. ${ }^{33-42}$ Of these, $30.5 \%(n=$ 114) were significant. Five studies analysed component summary scores. ${ }^{33,34,38,41,42}$ Physical component summary scores were reduced with weight gain in 22 of 27 analyses, 12 of which were significant. There were no analyses in which weight gain was associated with significantly improved physical component summary score. Mental component summary scores improved with weight gain in 14 of 27 analyses, 6 of which were significant. There were no analyses in which weight gain was associated with significantly reduced mental component summary score. Four 
studies analysed subscale scores. ${ }^{35,36,39,40}$ Physical subscales were predominantly reduced with weight gain. Mental subscales were mixed in their direction of association, except for vitality, which was predominantly reduced.

Results for weight gain via the predictive association are summarised in Table 3. Weight gain predicted lower physical component summary scores or physical subscales in three studies.$^{43,46,47}$ Weight gain predicted lower mental subscales In two studies $^{43,47}$ and was non-significantly associated with mental component summary scores in one study. ${ }^{46}$

Results for dose-responsivity are summarised in Supplementary Material Table S3. Of the ten studies examining categories representing greater and lesser degrees of weight gain, all showed physical HRQOL outcomes reduced in a dose-responsive manner with weight gain, particularly among women. ${ }^{35,36,38,40-43,45-47}$ Doseresponsivity of mental HRQOL outcomes with weight gain was inconsistent. Subgroup analyses stratified by baseline BMI category showed results for weight gain were similar among adults with normal weight, overweight and obesity (Supplementary Material Figure S1). Döring et $\mathrm{al}^{45}$ found weight gain predicted lower overall EQ-5D score for all BMI categories (Table 3). Stratification by sex showed reduced physical HRQOL with weight gain was similar for males and females (Supplementary Material Figure S3). Improved mental component summary scores with weight gain was only seen in females, however, there were fewer analyses for men. 


\section{Children}

Results for weight gain in children are summaries in Table 3. None of the identified studies examined weight gain via the change-on-change association in children. For the predictive association, weight gain predicted lower physical component summary scores or physical subscales in two studies. ${ }^{50,52}$ Weight gain predicted lower mental component summary scores in one study ${ }^{52}$ and was non-significantly associated with mental subscales in the other. ${ }^{50}$

This article is protected by copyright. All rights reserved. 

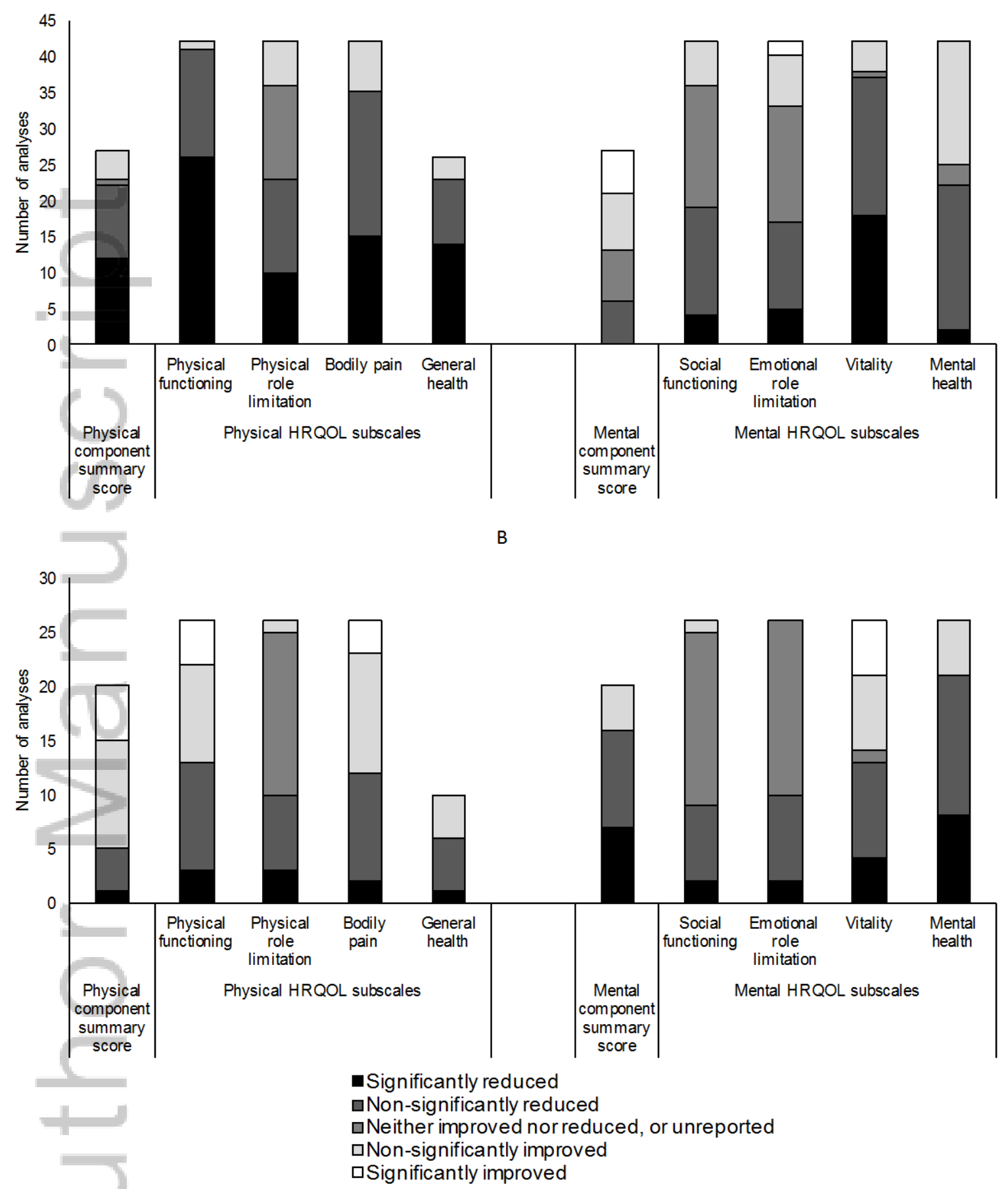

Figure 2 Tallied synthesis of 606 analyses for the association between weight gain (A) $(n=374)$ and weight loss (B) $(n=232)$ and changes in physical and mental component summary scores and subscales 


\section{Weight loss}

\section{Adults}

Results for weight loss in adults via the change-on-change association are summarised Figure 2B. Overall, from seven studies there were 232 analyses of the association between weight loss and change in HRQOL. ${ }^{33,36,38-42}$ Of these, $21.6 \%(n=50)$ were significant. Four studies analysed component summary scores. ${ }^{33,38,41,42}$ There were significant reductions and improvements in physical component summary scores with weight loss. Mental component summary scores were most often reduced. Three studies analysed subscale scores. ${ }^{36,39,40}$ There were significant reductions and improvements in physical subscales and the mental subscale of vitality with weight loss. Other mental subscales were most often reduced.

Results for weight loss via the predictive association are summarised in Table 3. In one study weight loss was not significantly associated with either physical or mental HRQOL at follow-up. ${ }^{43}$

Results for dose-responsivity are summarised in Supplementary Material Table S4. From the five studies examining categories representing greater and lesser degrees of weight loss, there was inconsistency in the dose-responsivity of both physical and mental HRQOL outcomes with weight loss. ${ }^{36,38,41,43,45}$

Subgroup analyses stratified by baseline BMI category indicated that in adults with overweight and obesity, weight loss was most often associated with improvements in physical HRQOL and vitality (Supplementary Material Figure S2). In adults with normal 
weight, weight loss was most often associated with reductions in physical and mental HRQOL (Supplementary Material Figure S2). Döring et $\mathrm{al}^{45}$ found weight loss among adults with overweight and obesity predicted lower overall ED-5Q score at follow-up (Table 3). Sex differences in HRQOL outcomes with weight loss were not reliably discernible because there were too few analyses in men (Supplementary Material Figure S4).

\section{Children}

Results for weight loss in children are summarised in Table 3. None of the identified studies examined weight loss via the change-on-change association in children. For the predictive association, one study found weight loss in children with obesity predicts higher physical component summary scores at follow-up, but was not significantly associated with mental component summary scores. ${ }^{49}$

\section{Weight change}

\section{Adults}

Results in adults for weight change as continuous variable are summarised in Table 3 . For the change-on-change association, weight change was significantly associated with changes in physical component summary scores in two studies. ${ }^{37,38}$ Only one study reported the direction of association as inverse. ${ }^{38}$ Weight change was directly associated with changes in mental component summary scores in one study ${ }^{38}$ and non-significantly associated in the other. ${ }^{37}$ For the predictive association, weight change was not significantly associated with either physical or mental HRQOL at follow up in one study. ${ }^{44}$ 


\section{Children}

Results in children for weight change as continuous variable are summarised in Table 3. For the change-on-change association, weight change was inversely associated changes in both physical component summary scores and one mental subscale in one study. ${ }^{48}$ For the predictive association, weight change was not significantly associated with physical and mental HRQOL at follow-up in one study. ${ }^{51}$

\section{Discussion}

In this systematic review we included 20 studies examining the longitudinal association between weight change and HRQOL in adults and children. For the change-on-change association in adults, we made four key findings:

1) Weight gain was consistently associated with a reduction in both physical HRQOL and the mental HRQOL subscale of vitality across all BMI categories and in both males and females, with demonstrated dose-responsivity.

2) Weight gain was inconsistently associated with changes in other mental HRQOL subscales, with associations comparatively less consistent in their direction; less often significant; and less often demonstrating dose-responsivity.

3) Weight loss among adults with overweight and obesity seemed to be associated with improved physical HRQOL and vitality, yet changes in other mental HRQOL subscales were inconsistent. 
4) Weight loss among adults of normal weight seemed to be associated with reductions in both physical and mental HRQOL.

Results for children; for the predictive association; and for weight change as a continuous variable were generally consistent with these findings.

\section{Comparison with existing research and interpretation}

Previous systematic reviews and meta-analyses of cross-sectional studies have similarly demonstrated a strong dose-responsive inverse relationship between weight and physical HRQOL in both adults and children. ${ }^{10,11,13,14,55}$ Secondary analyses of longitudinal intervention studies further show weight gain is associated with reduced physical HRQOL. ${ }^{56,57}$ The mechanism of this association is unknown. The physical burden of carrying additional weight may directly impede physical aspects of life, thereby reducing HRQOL. It may also result from the development of obesity-related co-morbidities, such as cardiovascular disease and osteoarthritis, and their subsequent negative HRQOL impacts, particularly in adults. ${ }^{58}$ Most included studies adjusted for baseline co-morbidities, however, only five adjusted for incident co-morbidities and it is possible not all comorbidities were accounted for. ${ }^{34,38-41}$ Furthermore, convincing evidence exists for a positive, dose-responsive association between physical activity, physical fitness and HRQOL. ${ }^{59-61}$ While several included studies adjusted for baseline levels, only Pan et al ${ }^{41}$ adjusted for changes in physical activity. While our key findings echo those of Pan et al ${ }^{41}$, it remains possible that changes in weight and physical HRQOL were partly mediated through changes in physical activity and fitness. This mechanism may be more prominent 
in children, as obesity-related co-morbidities, while possible in children, are more common in adulthood. ${ }^{3}$

As with our findings, existing literature on weight and mental HRQOL outcomes is inconsistent. One review in adults found mental HRQOL was significantly lower only with $\mathrm{BMI} \geq 40 \mathrm{~kg} / \mathrm{m}^{2}$ and was significantly higher in the overweight category. ${ }^{55}$ In children, one review $^{14}$ showed an inverse relationship between weight and overall mental HRQOL, while three reviews found only one PedsQL mental subscale was consistently lower at higher weights. ${ }^{10,11,13}$ Longitudinal intervention studies found weight gain to be variably associated with improved ${ }^{56,57}$ and reduced ${ }^{62}$ mental HRQOL. It is possible that weight and mental HRQOL are unrelated or only weakly related, with mental HRQOL only affected at extremes of weight change. Furthermore, generic HRQOL instruments used in these studies and in our review are less sensitive to weight than obesity-specific instruments, and may not detect subtle mental HRQOL changes with weight changes. ${ }^{21,62}$ Throughout most of human history increased weight has been considered a sign of health and prosperity. ${ }^{63}$ This is still true in many cultures today, which may further dilute negative impacts of weight gain on mental HRQOL or even account for observed improvements in mental HRQOL with weight gain. ${ }^{63}$

Previous reviews of weight loss interventions have similarly found improved physical HRQOL outcomes with weight loss among adults with overweight and obesity. ${ }^{15,16}$ It is possible those who experienced improved HRQOL with weight loss in our included studies were intentionally seeking treatment. People seeking treatment for obesity have lower HRQOL than the general population with obesity, and therefore stand to gain the most 
from weight loss treatment, limiting the generalisability of results from treatment-seeking populations. ${ }^{21}$ In these populations, it may also be exposure to the intervention itself which causes improved HRQOL independent of actual weight loss. ${ }^{17-20}$

The finding of reduced physical and mental HRQOL outcomes with weight loss among adults with normal may relate to underweight. Two studies combined participants with underweight and normal weight in one category. ${ }^{36,40}$ Adults with underweight have lower HRQOL than adults with normal weight. ${ }^{64}$ If adults of normal weight or underweight loose further weight, a reduced HRQOL should logically follow. The reduced HRQOL and weight

loss may also be result from the development of a disease, such as cancer. ${ }^{65}$ While we excluded studies, which included only participants with diagnosed illnesses, we were unable to differentiate between intentional weight loss from lifestyle modification or medical intervention and unintentional weight loss.

\section{Public health implications}

Our findings strengthen evidence for a causal association between weight and physical HRQOL. Reduced physical HRQOL with weight gain was consistent across studies and demonstrated dose-responsivity. However, given changes in weight and HRQOL occurred concurrently, we were unable to demonstrate temporality. Studies investigating bidirectional associations found associations with BMI as the predictor of HRQOL outcomes showed stronger dose-responsivity than reverse associations with HRQOL as the predictor of weight outcomes. ${ }^{34,66}$ This suggests weight as a causal factor for physical HRQOL outcomes. Further research involving multiple measurement intervals is needed to clarify the temporal relationship. 
Reduced physical HRQOL with weight gain may also be clinically important. Five point differences in SF-36 subscale scores and two to three point differences in summary scores are considered clinically important. ${ }^{23,67}$ Several included studies using the SF-36 reported clinically important reductions in physical HRQOL outcomes with weight gain. ${ }^{34-36,}$ $39-41,43,47$ These studies examined population samples resembling the general population. Clinically important reductions in physical HRQOL with weight gain are of public health concern when seen at the general population level.

Furthermore, our findings suggest weight loss may be unable to reverse the negative impacts of weight gain on HRQOL. Among the studies there was a lower proportion of significant associations for weight loss compared with weight gain, suggesting HRQOL is less strongly associated with weight loss. Several included studies found that persistent overweight or obesity was associated with even lower physical HRQOL outcomes than developing overweight or obesity. ${ }^{35,38,46,52}$ It may be difficult for weight loss to reverse the combined HRQOL effects of weight gain and the length of time spent with overweight or obesity. If this is true, public health approaches aimed at preventing weight gain are likely to have a greater positive impact on HRQOL than strategies designed to deal with obesity once it has fully developed. ${ }^{63}$

As with adults, we found reduced physical HRQOL with weight gain in children. These negative physical HRQOL impacts of weight gain in childhood are compounded by the persistence of childhood obesity into adulthood. ${ }^{68}$ This is of particular public health concern, given the rising prevalence of obesity among children. Thus our findings support the World Health Organization's position that more attention should be given to strategies 
aimed at preventing weight gain and obesity at the population level, and that prevention should target both children and adults. ${ }^{63}$ Prevention of weight gain from early in life may not only affect morbidity and mortality, but may also improve HRQOL in the general population. $^{3}$

\section{Strengths and limitations}

To the authors' knowledge, this is the first systematic review to examine the longitudinal association between weight gain and HRQOL. Our review also adds longitudinal information absent from previous reviews of cross-sectional studies. This temporal element allowed us to examine predictive and change-on-change associations for weight changes and HRQOL outcomes. These associations were examined in both children and adults.

At the study level, we decided to include only longitudinal observational studies, which are more prone to various types of bias. Our research question could not be answered with randomised control trials, as weight loss interventions do not provide information on weight gain and exposure to interventions themselves may cause HRQOL changes independent of weight loss. ${ }^{17-20}$ In the included studies, long follow-up times often led to considerable loss to follow-up, and the selection of healthier populations. The number of significant reductions and significant improvements in HRQOL may, therefore, be underand overestimated, respectively. Finally, two studies assessed self-reported weight change by recall, potentially introducing recall bias. ${ }^{39,47}$ 
At the review level, we searched for and included only the six most prevalent HRQOL instruments to reduce heterogeneity. We did not search for HRQOL synonyms, such as well-being, health status and utility. This may have inadvertently excluded some relevant studies measuring HRQOL with other instruments. The tallying synthesis was provided to summarise the number and direction of associations between weight change and HRQOL, but neglects the magnitude of weight and HRQOL changes. This synthesis was only possible for the change-on-change association in adults, as all studies used the SF-36 or SF-12. In the synthesis, there were fewer analyses of weight loss, reducing the reliability of our findings for weight loss. The synthesis was also not weighted by sample size and its internal validity is limited by the high degree of between-study heterogeneity in design, population characteristics, and statistical methods. In particular, differences in the definitions of weight change precluded a meta-analysis. Finally, we assumed unreported results to be non-significant. This was an attempt to include all negative results, though the assumption of non-significance may have caused misclassification.

The external validity of our findings may be limited. Studies contributing many analyses were overrepresented in the tallying synthesis. The study by Fine et $\mathrm{al}^{36}$ surveyed middleand older-aged predominantly white female nurses and contributed over one third of the tallied analyses. Additionally, all studies were from developed Western nations and just five studies were in children. Several studies indicate age, ethnicity, sex, and socioeconomic status may influence the relationship between weight and HRQOL. ${ }^{69-72}$ None of the included studies examined participants with underweight as a distinct category. Our findings may, therefore, be less generalisable to children and younger- 
adults; populations of lower socioeconomic status; people of non-white ethnicities; nonWestern or developing nations; people with underweight; and males. Notably, of the included studies that examined males and females separately, all reported weight change to be more strongly associated with HRQOL in females than males. ${ }^{34,35,38-40,43}$ The fewer analyses for men prevented us from concluding this definitively.

\section{Conclusions}

This review found a consistent, dose-responsive, and inverse relationship between weight gain and physical HRQOL and vitality in adults. Associations between weight gain and mental HRQOL were less consistent. For adults with overweight and obesity, weight loss may be associated with improved physical HRQOL and vitality. However, for adults of normal weight, weight loss may be associated with decreased physical and mental HRQOL. The relationships between weight change and HRQOL in children mimicked those in adults, but require further research to replicate findings. Since weight gain was more strongly associated with $\mathrm{HRQOL}$ than weight loss and weight gain is seen to have a detrimental impact on physical HRQOL from childhood, this review suggests public health preventative strategies for both adults and children offer the greatest potential to reverse the negative HRQOL impacts of the obesity epidemic.

\section{List of abbreviations}

$\mathrm{BMI}$ - body mass index

EQ-5D - Euroqol 5D 
HRQOL - Health-related quality of life

JKH - Johanna Katharina Hohls

$\mathrm{MJH}$ - Mark Joseph Hayes

PedsQL - Pediatric Quality of Life Inventory

QATOCCS - Quality Assessment Tool for Observational Cohort and Cross-Sectional

Studies

RM - Rebecca Muckelbauer

SF-12 - Short Form 12

SF-36 - Short Form 36

\section{References}

1

$\mathrm{Ng} \mathrm{M}$, Fleming $\mathrm{T}$, Robinson $\mathrm{M}$, et al. Global, regional, and national prevalence of overweight and obesity in children and adults during 1980-2013: a systematic analysis for the Global Burden of Disease Study 2013. Lancet. 2014;384(9945):766-81.

2 World Health Organization. General Assembly resolution 66/2. Political declaration of the high-level meeting of the General Assembly on the prevention and control of noncommunicable diseases. Sixty-sixth session of the General Assembly; 19th September. Geneva, Switzerland: United Nations; 2011.

3 Simmonds M, Burch J, Llewellyn A, et al. The use of measures of obesity in childhood for predicting obesity and the development of obesity-related diseases in 
adulthood: a systematic review and meta-analysis. Health Technol Assess. 2015;19(43):1336.

$4 \quad$ Aune D, Sen A, Prasad M, et al. BMI and all cause mortality: systematic review and non-linear dose-response meta-analysis of 230 cohort studies with 3.74 million deaths among 30.3 million participants. BMJ (Clinical research ed). 2016;353:i2156.

$5 \quad$ Fontaine KR, Barofsky I. Obesity and health-related quality of life. Obes Rev. 2001;2(3):173-82.

6 Guyatt GH, Feeny DH, Patrick DL. Measuring health-related quality of life. Ann Intern Med. 1993;118(8):622-9.

7 Mapes DL, Lopes AA, Satayathum S, et al. Health-related quality of life as a predictor of mortality and hospitalization: the Dialysis Outcomes and Practice Patterns Study (DOPPS). Kidney Int. 2003;64(1):339-49.

8 Tapp RJ, O'Neil A, Shaw JE, Zimmet PZ, Oldenburg BF. Is there a link between components of health-related functioning and incident impaired glucose metabolism and type 2 diabetes? The Australian Diabetes Obesity and Lifestyle (AusDiab) study. Diabetes Care. 2010;33(4):757-62.

$9 \quad$ Kroenke $\mathrm{CH}$, Kubzansky LD, Adler N, Kawachi I. Prospective change in healthrelated quality of life and subsequent mortality among middle-aged and older women. Am J Public Health. 2008;98(11):2085-91.

10 Griffiths LJ, Parsons TJ, Hill AJ. Self-esteem and quality of life in obese children and adolescents: a systematic review. Int J Pediatr Obes. 2010;5(4):282-304. 

and adolescents. Int J Obes. 2009;33(4):387-400. between body mass index and health-related quality of life among adults, assessed by the SF-36. Obesity. 2013;21(3):E322-7.

13 Sanders RH, Han A, Baker JS, Cobley S. Childhood obesity and its physical and psychological co-morbidities: a systematic review of Australian children and adolescents. Eur J Pediatr. 2015;174(6):715-46.

Ul-Haq Z, Mackay DF, Fenwick E, Pell JP. Meta-analysis of the association between body mass index and health-related quality of life among children and adolescents, assessed using the pediatric quality of life inventory index. J Pediatr. 2013;162(2):280-6.e1.

Warkentin LM, Das D, Majumdar SR, Johnson JA, Padwal RS. The effect of weight loss on health-related quality of life: systematic review and meta-analysis of randomized trials. Obes Rev. 2014;15(3):169-82.

16 Kroes M, Osei-Assibey G, Baker-Searle R, Huang J. Impact of weight change on quality of life in adults with overweight/obesity in the United States: a systematic review. Curr Med Res Opin. 2016;32(3):485-508.

17

Brito E, Patrick DL, Konopken YP, Keller CS, Barroso CS, Shaibi GQ. Effects of a diabetes prevention programme on weight-specific quality of life in Latino youth. Pediatr Obes. 2014;9(5):e108-11. 

of life and body mass index in overweight adult women with polycystic ovary syndrome during a lifestyle modification program. J Obstet Gynecol Neonatal Nurs. 2015;44(5):58799.

19 Rank M, Wilks DC, Foley L, et al. Health-related quality of life and physical activity in children and adolescents 2 years after an inpatient weight-loss program. J Pediatr. 2014;165(4):732-7.e2.

20 Fontaine KR, Barofsky I, Bartlett SJ, Franckowiak SC, Andersen RE. Weight loss and health-related quality of life: Results at 1-year follow-up. Eat Behav. 2004;5(1):85-8. 21 van Nunen AM, Wouters EJ, Vingerhoets AJ, Hox JJ, Geenen R. The health-related quality of life of obese persons seeking or not seeking surgical or non-surgical treatment: a meta-analysis. Obes Surg. 2007;17(10):1357-66.

22 Cohen J. A Coefficient of Agreement for Nominal Scales. Educational and Psychological Measurement. 1960;20(1):37-46.

23 Ware JE, Kosinski M, Keller S. SF-36 physical and mental health summary scales: A user's manual. Boston, MA: The Health Institute, New England Medical Centre; 1994. 24 Ware JE, Kosinski M, Keller SD. A 12-item Short-Form health survey: construction of scales and preliminary tests of reliability and validity. Med Care. 1996;34(3):220-33. 25 The EuroQol Group. EuroQol - a new facility for the measurement of health-related quality of life. Health Policy. 1990;16(3):199-208.

26 Varni JW, Seid M, Rode CA. The PedsQL: measurement model for the pediatric quality of life inventory. Med Care. 1999;37(2):126-39. 

Health Institute, New England Medical Centre; 1996. adolescents: a European public health perspective. Sozial- und Präventivmedizin. 2001;46(5):294-302.

29

Tayyem R, Ali A, Atkinson J, Martin CR. Analysis of health-related quality-of-life instruments measuring the impact of bariatric surgery: systematic review of the instruments used and their content validity. Patient. 2011;4(2):73-87.

30 Solans M, Pane S, Estrada MD, et al. Health-related quality of life measurement in children and adolescents: a systematic review of generic and disease-specific instruments. Value Health. 2008;11(4):742-64.

31 National Heart Lung and Blood Institute. Quality Assessment Tool for Observational Cohort and Cross-Sectional Studies: National Institutes of Health; [updated April 2014; cited 2016 April 6th]. Available from: http://www.nhlbi.nih.gov/health-

\section{pro/guidelines/in-develop/cardiovascular-risk-reduction/tools/cohort.}

32 Moher D, Liberati A, Tetzlaff J, Altman DG, The PG. Preferred reporting items for systematic reviews and meta-analyses: the PRISMA statement. PLoS Med. 2009;6(7):e1000097.

33 Batsis JA, Zbehlik AJ, Barre LK, Bynum J, Pidgeon D, Bartels SJ. Impact of obesity on disability, function, and physical activity: data from the Osteoarthritis Initiative. Scand J Rheumatol. 2015;44(6):495-502. 

obesity and health-related quality of life: evidence from the longitudinal AusDiab study. International Journal of Obesity. 2012;36(2):295-303.

35 de Hollander EL, Picavet HS, Milder IE, Verschuren WM, Bemelmans WJ, de Groot LC. The impact of long-term body mass index patterns on health-related quality of life: the Doetinchem Cohort Study. Am J Epidemiol. 2013;178(5):804-12.

Fine JT, Colditz GA, Coakley EH, et al. A prospective study of weight change and health-related quality of life in women. JAMA. 1999;282(22):2136-42.

37 Herpertz S, Muller A, Burgmer R, Crosby RD, de Zwaan M, Legenbauer T. Healthrelated quality of life and psychological functioning 9 years after restrictive surgical treatment for obesity. Surgery for obesity and related diseases : official journal of the American Society for Bariatric Surgery. 2015;11(6):1361-70.

38 Laxy M, Holle R, Doring A, Peters A, Hunger M. The longitudinal association between weight change and health-related quality of life: the KORA S4/F4 cohort study. Int J Public Health. 2014;59(2):279-88.

39 León-Muñoz LM, Guallar-Castillón P, Banegas JR, et al. Changes in body weight and health-related quality-of-life in the older adult population. Int $\mathrm{J}$ Obes. 2005;29(11):1385-91.

40 Milder IE, de Hollander EL, Picavet H, Verschuren W, de Groot LC, Bemelmans WJ. Changes in weight and health-related quality of life. The Doetinchem Cohort Study. Journal of Epidemiology and Community Health. 2014;68(5):471-7. 

of life: 2 cohorts of US women. Am J Epidemiol. 2014;180(3):254-62.

Williams LT, Young AF, Brown WJ. Weight gained in two years by a population of mid-aged women: how much is too much? Int J Obes (Lond). 2006;30(8):1229-33.

Burns CM, Tijhuis MAR, Seidell JC. The relationship between quality of life and perceived body weight and dieting history in Dutch men and women. Int J Obes.

2001;25(9):1386-92.

44 Connor AE, Baumgartner RN, Pinkston CM, Boone SD, Baumgartner KB. Obesity, ethnicity, and quality of life among breast cancer survivors and women without breast cancer: the long-term quality of life follow-up study. Cancer Causes Control. 2016;27(1):115-24.

45 Döring N, de Munter J, Rasmussen F. The associations between overweight, weight change and health related quality of life: Longitudinal data from the Stockholm Public Health Cohort 2002-2010. Prev Med. 2015;75:12-7.

46

Kozak AT, Daviglus ML, Chan C, Kiefe Cl, Jacobs DR, Jr., Liu K. Relationship of body mass index in young adulthood and health-related quality of life two decades later: the Coronary Artery Risk Development in Young Adults study. Int J Obes. 2011;35(1):13441.

Strandberg TE, Strandberg A, Salomaa VV, Pitkala K, Miettinen TA. Impact of midlife weight change on mortality and quality of life in old age. Prospective cohort study. Int J Obes. 2003;27(8):950-4. 

mental health problems in young children. Pediatrics. 2011;128(4):677-84.

49 Gopinath B, Baur LA, Burlutsky G, Mitchell P. Adiposity adversely influences quality of life among adolescents. J Adolesc Health. 2013;52(5):649-53.

50

Herman KM, Hopman WM, Craig CL. Are youth BMI and physical activity associated with better or worse than expected health-related quality of life in adulthood? The Physical Activity Longitudinal Study. Qual Life Res. 2010;19(3):339-49. 51 Parkinson KN, Adamson AJ, Basterfield L, Reilly JK, Le Couteur A, Reilly JJ. Influence of adiposity on health-related quality of life in the Gateshead Millennium Study cohort: longitudinal study at 12 years. Arch Dis Child. 2015;100(8):779-83.

52 Wake M, Canterford L, Patton GC, et al. Comorbidities of overweight/obesity experienced in adolescence: Iongitudinal study. Arch Dis Child. 2010;95(3):162-8. 53 Hays RD, Sherbourne CD, Mazel RM. The RAND 36-Item Health Survey 1.0. Health Econ. 1993;2(3):217-27.

Landis JR, Koch GG. The measurement of observer agreement for categorical data. Biometrics. 1977;33(1):159-74.

55 Ul-Haq Z, Mackay DF, Fenwick E, Pell JP. Meta-analysis of the association between body mass index and health-related quality of life among adults, assessed by the SF-36. Obesity (Silver Spring, Md). 2013;21(3):E322-7.

56 Müller-Nordhorn J, Muckelbauer R, Englert $\mathrm{H}$, et al. Longitudinal association between body mass index and health-related quality of life. PLoS ONE 2014;9(3):e93071. 

between weight change and quality of life in a community-based population: a prospective cohort study. Eur J Public Health. 2013;23(2):285-90.

58 Heo M, Allison DB, Faith MS, Zhu S, Fontaine KR. Obesity and quality of life: mediating effects of pain and comorbidities. Obes Res. 2003;11(2):209-16.

59 Bize R, Johnson JA, Plotnikoff RC. Physical activity level and health-related quality of life in the general adult population: a systematic review. Prev Med. 2007;45(6):401-15. 60 Feeny D, Garner R, Bernier J, et al. Physical activity matters: associations among body mass index, physical activity, and health-related quality of life trajectories over 10 years. J Phys Act Health. 2014;11(7):1265-75.

61 Morales PF, Sanchez-Lopez M, Moya-Martinez P, et al. Health-related quality of life, obesity, and fitness in schoolchildren: the Cuenca study. Qual Life Res. 2013;22(7):1515-23.

Kolotkin RL, Norquist JM, Crosby RD, et al. One-year health-related quality of life outcomes in weight loss trial participants: comparison of three measures. Health Qual Life Outcomes. 2009;7:53.

World Health Organization. Obesity: preventing and managing the global epidemic. Geneva: 2000. life in the older population: results from the German KORA-Age study. Health Qual Life Outcomes. 2011;9(1):1-10. 

assessment criteria with health-related quality of life in patients with advanced colorectal carcinoma. Eur J Cancer Care. 2012;21(4):505-16.

66 Jansen PW, Mensah FK, Clifford S, Nicholson JM, Wake M. Bidirectional associations between overweight and health-related quality of life from 4-11 years: Longitudinal Study of Australian Children. Int J Obes. 2013;37(10):1307-13.

Ware JE, Snow KK, Kosinski M, Gandek B. SF-36 health survey manual and interpretation guide. Boston, MA: The Health Institute, New England Medical Centre; 1993.

68 Singh AS, Mulder C, Twisk JW, van Mechelen W, Chinapaw MJ. Tracking of childhood overweight into adulthood: a systematic review of the literature. Obes Rev. 2008;9(5):474-88.

69 Zabelina DL, Erickson AL, Kolotkin RL, Crosby RD. The effect of age on weightrelated quality of life in overweight and obese individuals. Obesity (Silver Spring). 2009;17(7):1410-3.

70 Garner RE, Feeny DH, Thompson A, et al. Bodyweight, gender, and quality of life: a population-based longitudinal study. Qual Life Res. 2012;21(5):813-25.

71 Bentley TGK, Palta M, Paulsen AJ, et al. Race and gender associations between obesity and nine health-related quality-of-life measures. Qual Life Res. 2011;20(5):665-74. 72 Minet Kinge J, Morris S. Socioeconomic variation in the impact of obesity on healthrelated quality of life. Soc Sci Med. 2010;71(10):1864-71. 


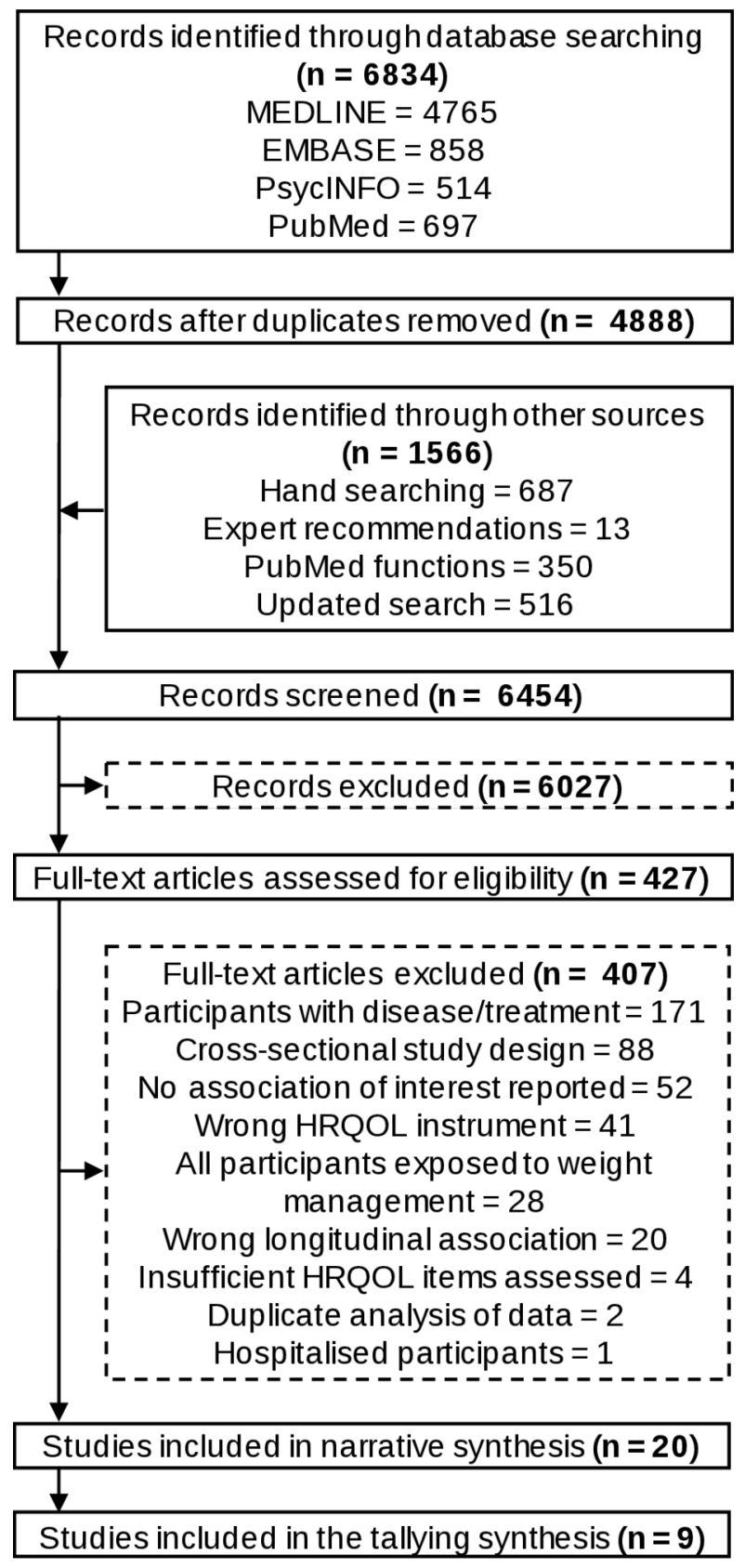

obr_12595_f1.eps 
A

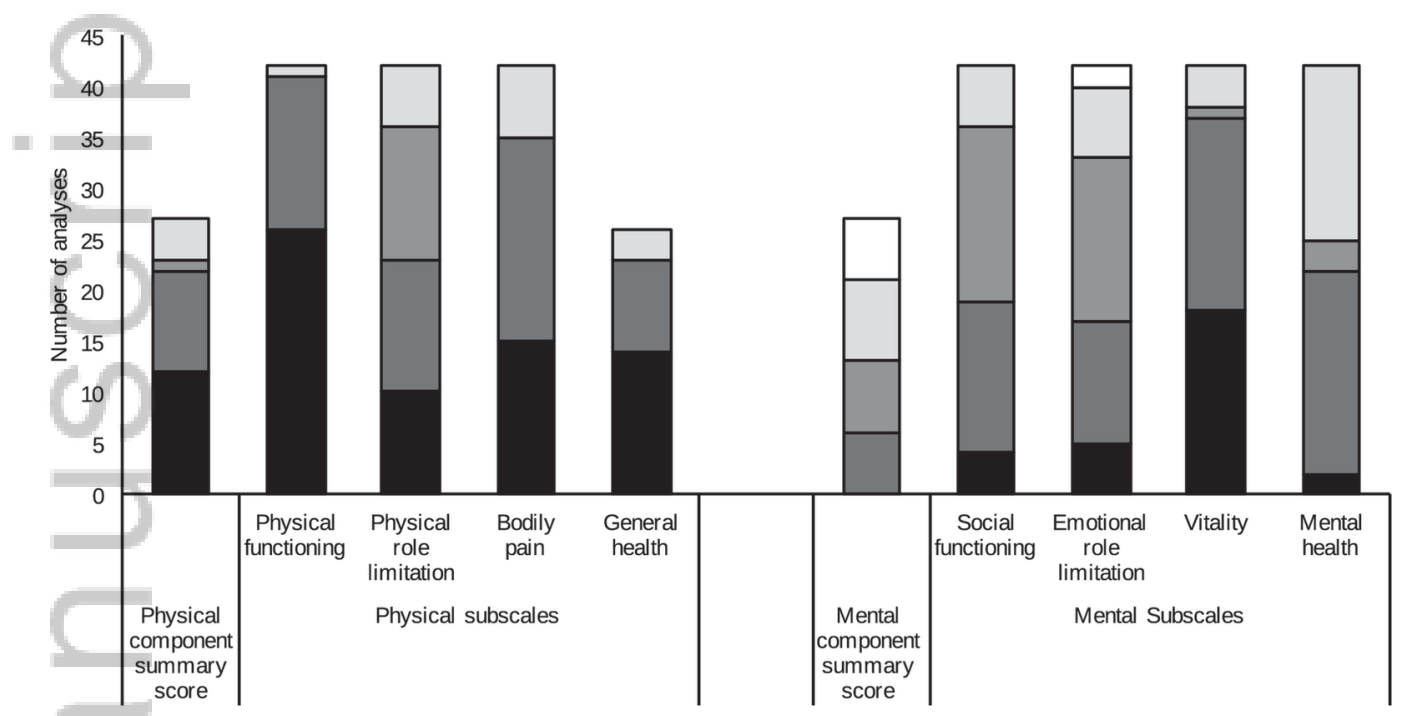

B

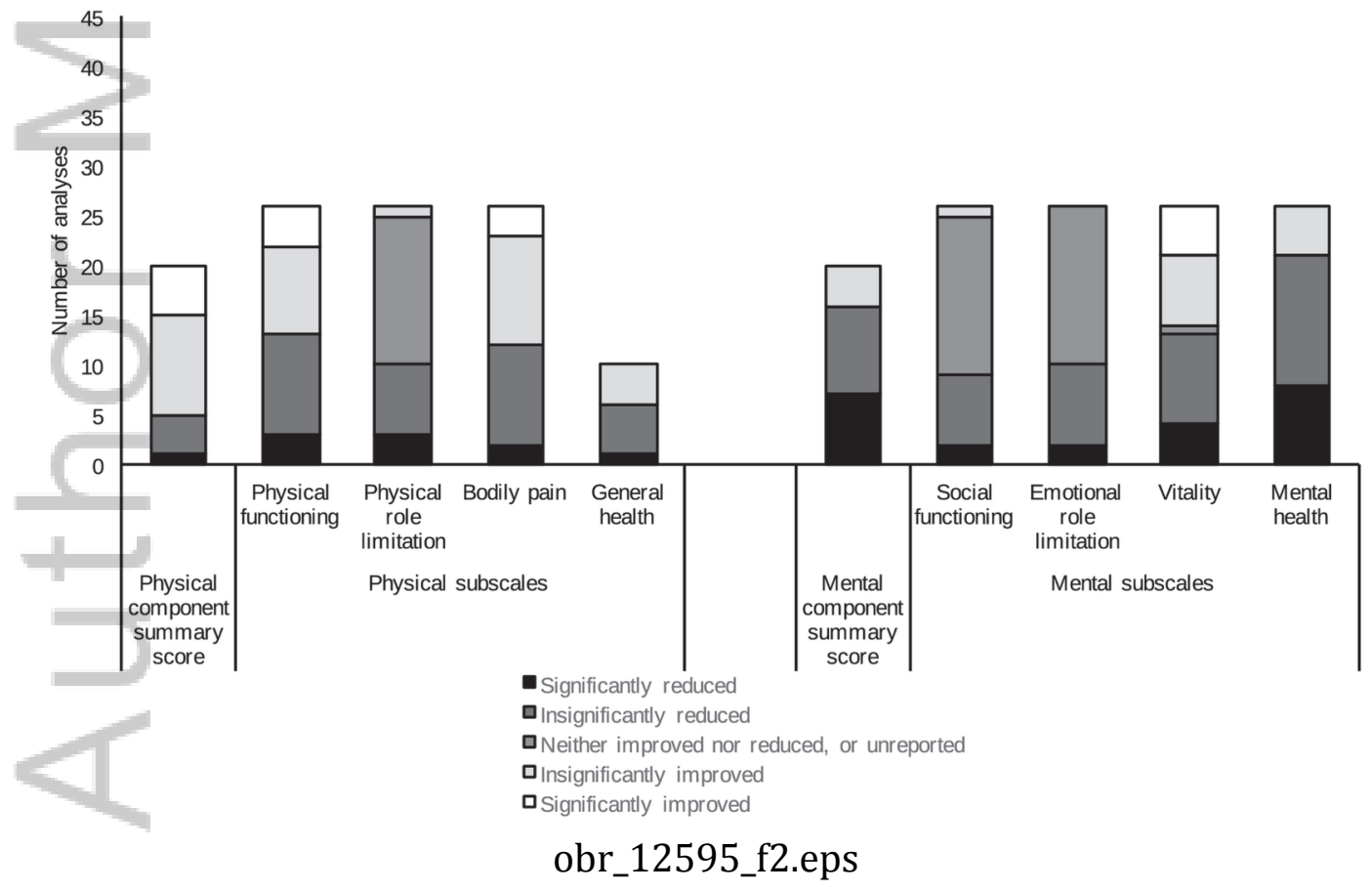

This article is protected by copyright. All rights reserved. 


\section{University Library}

\section{- M M N E R VA A gateway to Melbourne's research publications}

Minerva Access is the Institutional Repository of The University of Melbourne

Author/s:

Hayes, M;Baxter, H;Mueller-Nordhorn, J;Hohls, JK;Muckelbauer, R

Title:

The longitudinal association between weight change and health-related quality of life in adults and children: a systematic review

Date:

2017-12-01

Citation:

Hayes, M., Baxter, H., Mueller-Nordhorn, J., Hohls, J. K. \& Muckelbauer, R. (2017). The longitudinal association between weight change and health-related quality of life in adults and children: a systematic review. OBESITY REVIEWS, 18 (12), pp.1398-1411. https:// doi.org/10.1111/obr.12595.

Persistent Link:

http://hdl.handle.net/11343/293549 